\title{
Gastric ulcer and traditional Chinese medicine
}

\author{
Huan Xu ${ }^{1 \#}$, Huan Yao ${ }^{1 \#}$, Zhong Jiang ${ }^{1 \#}$, Xiao Wu1, Zixian Chen ${ }^{2}$, Wangming $\mathrm{Hu}^{1}$, Lingling Zhang ${ }^{1}$, Bing Liang ${ }^{1}$ and Yong Wang ${ }^{1 *}$ \\ ${ }^{1}$ Chengdu University of Traditional Chinese Medicine, College of Basic Medicine, Chengdu, China \\ ${ }^{2}$ Chengdu University of Traditional Chinese Medicine, School of sports, Chengdu, China \\ \#Equally contributed to this article
}

\begin{abstract}
Gastric Ulcer is a common type of peptic ulcers. Gastric Ulcer is characterized by injury of gastric mucosa. The common causes are including infection of bacteria Helicobacter Pylori, long-term use of nonsteroidal anti-inflammatory drugs. Some other contributors are included tobacco smoking, excessive drinking, spacy food and stress from modern life. Research also demonstrated that aging, hereditary background and underlying medical conditions could accelerate gastric ulcer development. The most common symptom of gastric ulcer is burning pain. Gastric ulcer patient also exhibited other symptoms, including poor appetite, weight loss, belching, melena, bloating and abdominal fullness, nausea and copious vomiting. Gastric ulcer is not a leading cause of mortality and morbidity. However, if untreated or inappropriated treatment, gastric ulcer would result in upper gastrointestinal bleeding, gastric perforation, pyloric obstruction and cancer. The treatment for gastric ulcers mainly includes gastric acid production inhibition, antagonistic cholinergic receptor, antagonistic $\mathrm{H} 2$ receptor, proton pump inhibitor, anti-Helicobacter pylori, and gastric mucosal protective drugs. However, due to low efficacy and high risk of side effects, its necessary to develop novel and efficient pharmacologic strategies for Gastric Ulcer treatment. Our previous study demonstrated that gastric vascular homeostasis is critical in regulating gastric ulcer development. In this study, we literate that common causes for gastric ulcer, the prevention and treatment for gastric ulcer. More important, we also demonstrated that Traditional Chinese medicine (TCM) processes great potential and advantage in treating gastric ulcer, especial for chronic and recurrent gastric ulcer.
\end{abstract}

\section{Introduction}

Peptic ulcer is a common digestive system disease, including duodenal ulcer and Gastric Ulcer. Gastric Ulcer is the most common gastrointestinal disorder of upper digestive tract. Gastric Ulcer is a benign lesion with multiple etiologies, mainly caused by injuring of gastric mucosa, including infection of bacteria Helicobacter Pylori, long-term use of nonsteroidal anti-inflammatory drugs [1]. Some other contributors are included tobacco smoking, excessive drinking, spacy food and stress from working [2]. Some research also demonstrated that aging, hereditary background and underlying medical conditions could accelerate the development of gastric ulcer [3]. The most common symptoms of gastric ulcer are burning pain that happened within upper abdomen. Other symptoms of gastric ulcer are including poor appetite, weight loss, belching, melena, bloating and abdominal fullness, nausea and copious vomiting. Gastric ulcer is not a leading cause of mortality and morbidity [4]. However, if untreated, gastric ulcer would result in upper gastrointestinal bleeding, gastric perforation, pyloric obstruction and cancer.

The treatment for gastric ulcers mainly include acid production inhibition, antagonistic cholinergic receptor, antagonistic $\mathrm{H} 2$ receptor, proton pump inhibitor, anti-Helicobacter pylori, and gastric mucosal protective drugs such as sulphoaluminium and bismuth agents [5-7]. An army of research demonstrated that development of gastric ulcer was due to damage of gastric mucosal, which caused by multiple stresses [8].

Gastric mucosal comprises epithelial lining, submucosa and muscularis externa. Studies indicate that gastric mucosal is an important barrier for gastric self-protection, and those barriers are including mucus bicarbonate barrier, epithelial barrier, continuous epithelial renewal, mucosal microcirculation blood flow, mucosal microvascular endothelial barrier [9].

\section{The causes of Gastric Ulcer}

Gastric mucosal damaging was close related to gastric ulcer development [10]. A series causes are associated with gastric ulcer development. The best known causes for gastric ulcer development are Helicobacter pylori infection and long-tern use of nonsteroidal antiinflammatory drugs [11]. Studies indicated that heavy tobacco smoking, excessive drinking, too much spacy food can also cause gastric ulcer [12]. some other causes may not cause gastric ulcer directly, however, which can make people more susceptible to gastric ulcer or make worse of gastric ulcer. The aging people who always accompany by insufficiency of sleep, lack of exercise is more susceptible to gastric ulcer [13]. Research demonstrated that peoples suffered from long-term mental stress, mood swings, anxiety and depression due to fast pace of modern life are more likely developing gastric ulcer [14]. Development of gastric ulcer are also associated with hereditary background, underlying medical conditions, for example, coronary cardiovascular disease, gastric carcinoma, gastric inflammation [15]. Deference of geographical environment, climate changing and season changing could accelerate the development of gastric ulcer [16].

\section{Helicobacter pylori infection}

The most common cause of gastric ulcer is infected by bacterium Helicobacter pylori [17]. Helicobacter pylori was first reported by

${ }^{\star}$ Correspondence to: Wang Y, Ph.D, Basic Medical College, Cheng Du University of Traditional Chinese Medicine, Chengdu, China E-mail: yongwang1008@hotmail. com/wangyong@cdutcm.edu.cn

Key words: gastric ulcer, gastric mucosal, vascular homeostasis, microenvironment, traditional chinese medicine

Received: January 29, 2021; Accepted: February 05, 2021; Published: February 08,2021 
Warren and Marshall in1983 [18,19]. Helicobacter pylori is a Gramnegative bacterial pathogen that selectively colonizes within gastric epithelium. It is urease, catalase, oxidase positive, spiral shaped, and possesses 3 to 5 polar flagella that are used for motility. Studies demonstrated that Helicobacter pylori infection plays an important role in development of pathogenic chronic gastritis, peptic ulcers and gastric carcinoma. Helicobacter pylori expresses inflammatory protein adhesion (OipA) and blood group antigen adhesion (BabA), which promotes attachment to gastric epithelium characterized by binding to variable number tandem repeat sequence (VNTR) oligosaccharides or Type Le b and Type H 1 epitopes. Helicobacter pylori also expresses virulence factors, including $\mathrm{Cag} A$ and $\mathrm{PicB}$, which induces gastric mucosal inflammation. Helicobacter pylori can inhibit hydrogen potassium ATPase, whereas actives calcitonin gene-related peptide sensory neurons, which induces somatostatin secretion to suppress acid production $X^{\prime}$. Urease secreted by Helicobacter pylori can create an alkaline environment, which is propitious to its survival (Figure 1).

\section{Long-tern use of nonsteroidal anti-inflammatory drugs (NSAID)}

Long-term use of nonsteroidal anti-inflammatory drugs (NSAIDs), such as ibuprofen and naproxen sodium, is another major cause for gastric ulcer development [20,21]. NSAIDs are a generic term for many chemically distinct drugs. NSAIDs, such as aspirin, indomethacin and ibuprofen, are widely used in treatment for pain, arthritis, cardiovascular diseases. More recently, NSAIDs are used for prevention of colon cancer and Alzheimer disease. However, NSAIDs produce gastroduodenal ulcers in about $25 \%$ of users (bleeding and/ or perforations) and delay ulcer healing. NSAIDs can inhibit COX activity to reduce PGs production, including the relaxant PGI2/PGE2/ PGD2 and contractile TXA2/PGF2 $\alpha[22,23]$. Most NSAIDs are acidic in aqueous solution, whereas their $\mathrm{pH}$ values are higher than that of hydrochloric acid secreted from parietal cells. NSAIDs, therefore, move into mucosal cells because of a $\mathrm{pH}$ gradient that favor their uptake when gastric acid is secreted into the lumen (Figure 1).

\section{Other causes}

Multiple causes are contributed to gastric ulcer development. Difference of life styles, for example, tobacco smoking, excessive drinking, spacy food, are causative factors for gastric ulcer disease [24]. Too much stress resulting in over anxious from working due to fast pace of modern life and sleep insufficiency can lead to gastric ulcer [14]. Some research also indicates that aging, lack of exercise can make worse of gastric ulcer [13]. People with special hereditary background are more likely disturbed by gastric ulcer [3]. The underlying medical conditions, including stomach, coronary heart diseases, could accelerate the development of gastric ulcer [15].

\section{The damage of gastric mucosal results in gastric ulcer development}

Gastric mucosa is the most superficial layer of gastric tissue. It is composed of the upper cortex, lamina propria, and mucosal muscularis. Multiple causes are contributed to gastric ulcer development due to gastric ulcer damaging. Gastric Ulcer is characterized by ischemia and necrosis of gastric mucosa and rupture of gastric mucosa, which mainly caused by increased secretion of gastric acid and pepsin that caused by various pathological factors [25].

Gastric mucosa mainly relies on several layers of barriers to protect and maintain gastric mucosa from damaging [26,27]. Mucous bicarbonate barrier contains mucus layer, bicarbonate and immunoglobulin etc. It plays a pivotal role in preventing gastric ulcer development by enhanced gastric mucosa acid resistance. Studies shown that mucous bicarbonate barrier can prevent gastric mucosa damage by inhibiting reverse diffusion hydrogen ion to stomach cavity mucous membrane, and slow down hydrogen ions diffusion by

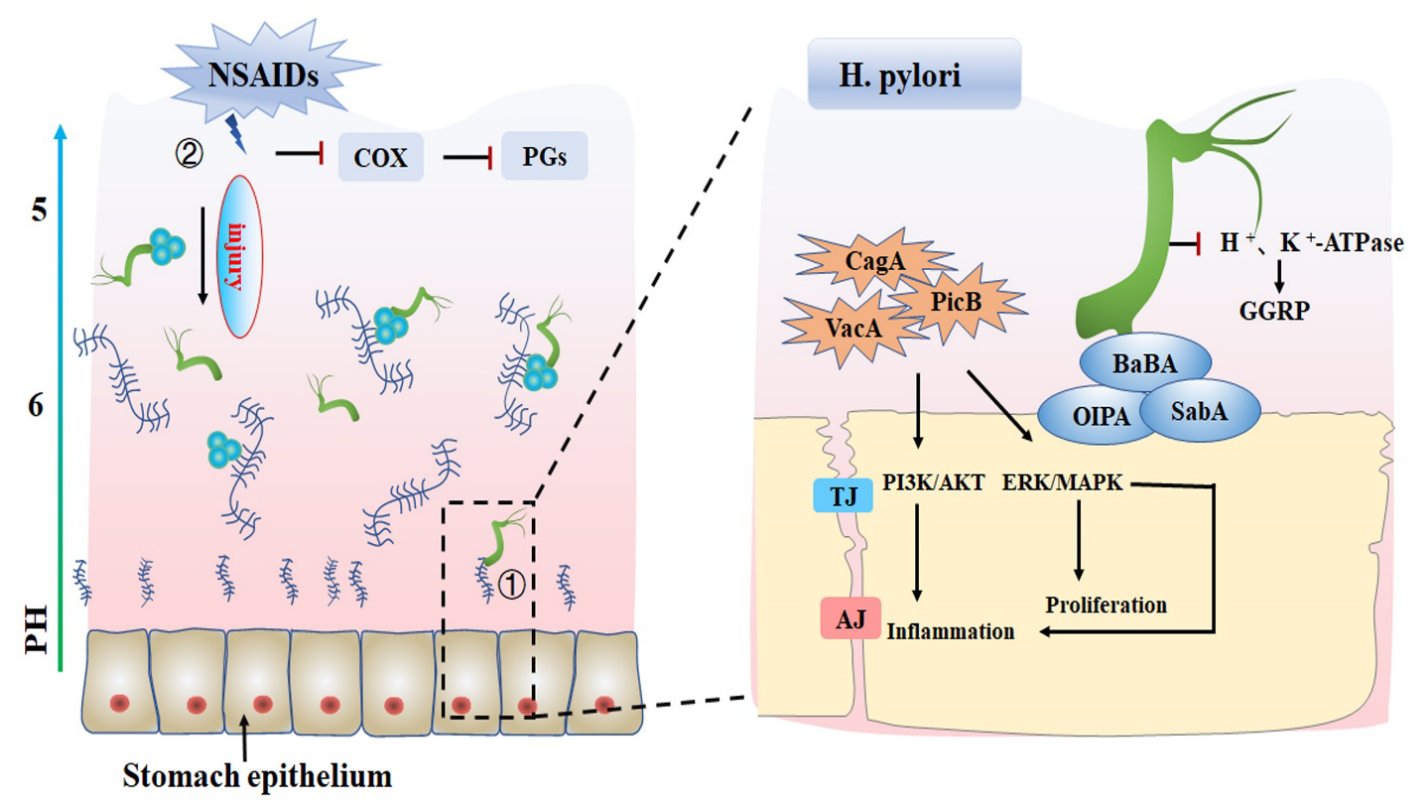

Figure 1. The common causes of gastric ulcers. (1) Helicobacter pylori infection leads to gastric ulcer. Helicobacter pylori expresses BABA, OIPA, SABA which promote epithelial cell adhesion. Helicobacter pylori can also express virulence factors such as CagA, VacA, and PicB, which can induce gastric mucosal inflammation through PI3K/AKT and ERK/MAPK signaling pathways. (2) Long-term use of NSAIDs can inhibit COX activity, reduce PG synthesis, promote gastric acid secretion, and damage gastric mucosa. TJ: Tight junction, AJ Adhesive junction, ERK: Extracellular regulated protein kinase, MAPK: Mitogen-activated protein kinase, GGRP: Calcitonin gene-related peptide, COX: Cycoxidase, PG: Prostaglandin, ERK: Extracellular regulated protein kinase, MAPK: Mitogen-activated protein kinase, BaBA: Blood group antigen binding adhesive, SaBA: Sialic acid binds to the adhesive, OIPA: Outer membran inflammatory proteins, CagA: Cytotoxin associated gene A, VacA: vacuolating cytotoxin A 
neutralize gastric acid environment. Mucous bicarbonate barrier is very important for gastric mucosa to maintain acid-base homeostasis.

Epithelial barrier is formed by tightly connected epithelial cells. Epithelial barrier secretes mucin and bicarbonate to maintain mucous bicarbonate barrier. It synthesizes and secretes three-foliated factor family peptide (TFF), which plays a critical role in maintain integrity of gastric surface in health and disease conditions by enhance gastric mucosal defense and promoting gastric mucosal repairing. Epithelial can also synthesizes and secretes prostaglandin (PG), which critical in regulating gastric inflammatory response after gastric injury.

Gastric mucosal microcirculation environment can provide nutrients, oxygen and some other regulators to maintain gastric mucosa homeostasis. Gastric mucosal microcirculation was regulated by nervous system. When gastric mucosa is stimulated by sensory afferent nerve, calcitonin gene-related peptide (CGRP) is released, which can increase blood flow, and also inhibit gastric acid secretion. Gastric mucosal microcirculation environment plays a pivotal role in regulating gastric mucosal inflammatory response, as well as promoting gastric ulcer recovering.

Continuous gastric mucosa epithelial renewal is also critical to maintain mucosal integrity. Under normal conditions, the integrity of gastric mucosa is maintained by synergistic effecting between mucus bicarbonate barrier, epithelial barrier, Gastric mucosal microcirculation environment and continuous epithelial renewal. The corruption of homeostasis within gastric mucosal leads to gastric ulcer development (Figure 2).

\section{Gastric mucosal vascular homeostasis is critical in reg- ulating gastric ulcer development}

Gastric mucosal microvessels are located within lamina propria. Our previously studies demonstrated that maintain gastric vascular homeostasis is critical in regulating gastric ulcer development [28]. The appropriated microenvironment associated vascular homeostasis is not only critical in protecting gastric mucosal form injury damage, but also important in promoting gastric mucosal repairing after injury [29].

\section{Intact vascular morphology Structure is critical in maintain environment homeostasis within gastric mucosal}

Gastric mucosal vessels are composed of endothelialcells (EC) and pericytes adherent to basement membrane. Response to stimulation, endothelial cells secrete a series of mediators to modulate function of vascular smooth muscle, such as vasoconstrictor endothelin, leukotriene, vasodilator nitric oxide (NO), prostacyclin 2 (PGI2). Endothelial cells can biosynsynthesis and release regulators, such as NO, PGI2, prostaglandin (PGE2), carbon monoxide (CO), plasminogen activator (t-PA), vascular endothelial growth factor (VEGF) and basic fibroblast growth factor (b-FGF) to maintain integrity of gastric mucosal [30,31]. These mediators reduce activation of platelets and white blood cells, prevent thrombosis, promote thrombolysis, maintain tissue perfusion, and protect microvascular walls from acute damage. Intact vascular morphology Structure is critical in maintain environment homeostasis within gastric mucosal, which can resistance to stimulation of gastric mucosal injury stresses.

\section{Interruption of gastric mucosal blood flow triggers or worsen gastric ulcer}

When exposed to various of injury stresses, or enhanced gastric acid invasion, gastric mucosal blood flow increases rapidly [32]. This hyperemia response mediated by sensory afferent nerves. Under stress, sensory nerves can release neurotransmitters, such as calcitonin generelated peptide (CGRP) and substance P. CGRP plays a gastric mucosal protective role through regulating $\mathrm{NO}$ formation. The vasodilation of submucosal vessels regulated by $\mathrm{NO}$ was also favored repairing of injury gastric mucosal. Study demonstrated that interrupted sensory afferent nerve can significantly inhibit hyperemia response. Disturbed gastric mucosal blood flow triggers or worsen gastric ulcer.

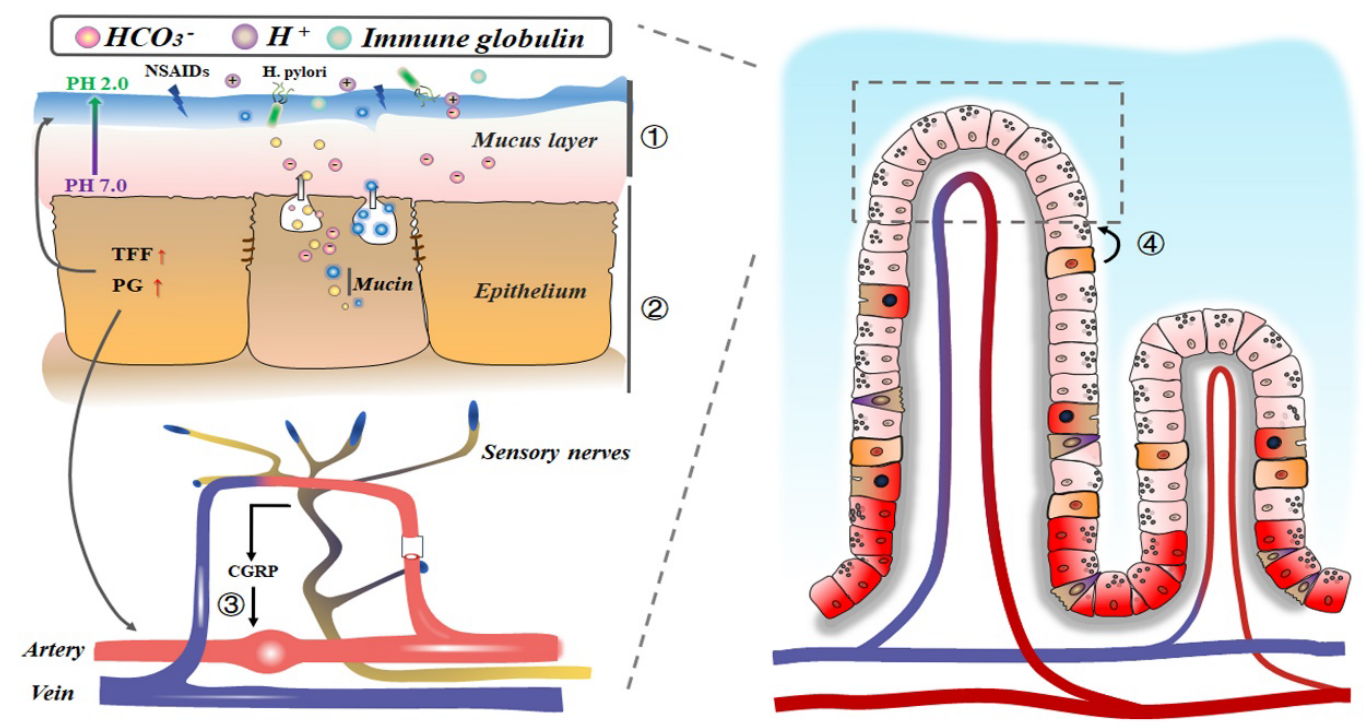

Figure 2. Gastric mucosa injury results in gastric ulcer. (1) gastric mucosal layer can slow down the diffusion rate of hydrogen ions, reduce the damage of hydrogen ions to gastric mucosa, and maintain the acid-base homeostasis of gastric mucosa; (2) Epithelial barrier can secrete mucin and bicarbonate, synthesis and secrete PG, TFF, and play a role in resistance to harmful substances. (3) Gastric mucosa microcirculation can provide nutrients and oxygen, maintain acid-base balance. When gastric mucosa is exposed to stimulators, sensory afferent nerve within gastric mucosal releases calcitonin CGRP, which can increase blood flow, inhibit gastric acid secretion. (4) Continuous renewal of gastric epithelial after gastric mucosal damage can facilitate complete repair of the gastric ulcer. PG: Prostaglandin, TFF: Trefoil factor family 


\section{Abnormal activation of vascular endothelial cell leads to imbalance of gastric mucosal homeostasis}

The mucosal vascular barrier is maintained by two types of junctions: tight junction and adhesion junction. Tight junctions include tight junction proteins Claudins, Occludins and adhesion molecules. While adhesion junctions include catenin, cadherin, mainly VE-cadherin. In response to injury stresses, endothelial cells can be activated and make change of structures, for example, losing of tight junction stimulated by histamine or 5-hydroxytryptamine, and leave a gap between adjacent endothelial cells at the site of vascular endothelial cadherin adhesion. These changes resulted in increased vascular endothelial permeability which can cause tissue edema, inflammation due to protein and liquid leakage. The activation of endothelial cells can be regulated by binding of VEGF to the receptor and activation of Src, ERK, JNK and PI3K/ AKT pathways [33]. Abnormal activation of vascular endothelial cell leads to imbalance of gastric mucosal homeostasis, which is tighten associated with gastric mucosal inflammation.

\section{Gastric mucosal vascular injury results in disturbing of} mucosal microenvirenmental homeostasis

The injury of microvascular endothelium leads to rupture of microvascular, exudation of plasma and red blood cells, which further interrupts blood flow and causes platelet aggregation and fibrin precipitation $[28,29]$. This process not only reduces oxygen and nutrient supplement, but also causes further damage to glandular epithelial cells by toxic substances. Deposition of fibrin on vascular endothelium plays an important role in regulating microvascular injury and repairing. On the one hand, fibrin-fibrinogen lysis products play a role in regulating tissue edema formation, endothelial cell retraction. On the other hand, fibrin-fibrinogen cleavage products can also induce expression of several endothelial cell-derived factors, such as intercellular adhesion molecule-1 (ICAM-1) and VCAM-1, Which leads to leukocyte aggregation, extravasation, and eventually leads to gastric ulcer development.

\section{Extracellular matrix remodeling is critical in maintain gastric mucosal vascular homeostasis}

Remodeling of extracellular matrix (ECM) is initiated when mucosal vascular endothelial cells exposed to injury factors, as well as in stage of tissue repair and neovascularization. Changes in vascular permeability results in macromolecular serum proteins leakage. Endothelial cells be activated in response to stimulation of injury factors or inflammatory factors, which can increase vascular permeability characterized by enhanced leukocyte infiltration, temporary matrix precipitation, and also promote angiogenesis in later stage of injury. During gastric ulcer repair stage, proliferation of vascular endothelial cells is enhanced through increased VEGF expression. Studies indicated that matrix metalloproteinases (MMP)-3 and 9 were significantly increased in indomethacin induced chronic Gastric Ulcer mice. MMP-9 expression was also detected in acetic acid-induced and ethanol-induced chronic and acute Gastric Ulcer [34,35]. Research demonstrated that increased expression of MMP is correlated with severity of gastric ulcer. These data indicated that extracellular matrix remodeling plays an important role in regulating pathogenesis of gastric ulcer through maintain gastric mucosal vascular homeostasis.

\section{TCM widely used in gastric ulcer treatment}

Gastric ulcer is not a leading cause of mortality and morbidity. Although remarkable progresses have been made in treatment of gastric ulcer recently. However, there is still no reasonable treatment for gastric ulcers, especially for chronic gastric ulcer and recurrent gastric ulcer. Our previous study demonstrated that Andrographolide plays a crucial role in maintaining gastric vascular homeostasis during gastric ulcer development through regulating vascular endothelial cell glycolytic pathway [28]. Actually, traditional Chinese medicine has been widely used in treating variety of diseases, including gastric ulcer [36].

\section{TCM relieves gastric ulcer burning pain symptom}

Majority of TCM are obtained from the nature world. Four properties of TCM are including Cold, Hot, warm and Cool. A variety of stresses from internal or external of human body can cause imbalance between yin and yang. The stresses processed heat characteristics can lead to multiple diseases, including gastric ulcer, which characterized by burning pain. The Cold and Cool properties of some TCMs can take away heat pathological stresses from circulated blood, and relieve burning pain symptom of gastric ulcer. Those TCMs are including scutellaria baicalensis georgi, coptis chinensis franch and phellodendron amurense rupr.

\section{TCM regulates production of gastric acid}

Too much gastric acid production can damage gastric mucosal, which leads to gastric ulcer development. Studies indicated that some TCMs, if combining with other treatments, can greatly decrease production of gastric acid. It has been reported that Solanum nigrum can decreased gastric acid concentration. However, those TCMs probably can not inhibit gastric acid production directly, it possible through bacteriostasis, including Helicobacter pylori and some other intestinal pathogenic bacteria [37]. Those TCMs are including Lonicera japonica thund, hounttuynia cordata thumb, Pulsatilla chinensis regel etc.

\section{TCM suppresses gastric mucosal inflammation}

A variety of stresses can induce gastric ulcer development, which was associated with inflammatory response. An accumulated studies demonstrated that some TCMs pay an important role in treatment of inflammatory diseases [38]. Some TCMs can promote secretion of endogenous of cortisol through regulating the activity of hypothalamus-pituitary-adrenal cortex axis. Research indicated that some TCMs are critical in Suppressing the synthesis and secretion of Prostaglandin E2 (PGE2), Inhibiting the activity of Cyclooxygenase, Impairing the production and release of thromboxane, preventing production of leukotriene B4, Histamine, 5-hydroxytryptamine. The anti-inflammatory function of some TCMs is important for in relieving symptoms of gastric ulcer and promoting gastric ulcer recovering.

\section{TCM improves gastric vascular microenvironment}

Some studies have found that some TCMs can promote gastric mucosal repairing through stimulating gastric mucosal endothelial cell proliferation and promoting angiogenesis. Our previous study demonstrated that andrographolide regulates glycolysis pathway of vascular endothelial cells and plays an important role in maintaining gastric vascular homeostasis during gastric ulcer development [28] Tanshinone II A attenuates vascular remodeling through klf4 mediated smooth muscle cell phenotypic switching [39].

\section{TCMs relieves oxidative stress}

Research demonstrated that Oxidative stress can trigger gastric ulcer or make gastric ulcer worsen 40. Studies shown that Rhizoma coptidis and Atractylodes rhizoma can significantly improve serum glutathione peroxidase (GPx), superoxide dismutase (SOD), catalase (CAT), while greatly reduce serum malondialdehyde level. 
Taken together, this study literatured the common causes that lead to gastric ulcer, as well as the critical roles of mucosal microenvironment regulated by vascular homeostasis in regulating gastric ulcer devolopment. In this study, we also focus on the therapytic roles of TCM in treatment of gastric ulcer. Long term clinicle usage of TCM indicated that TCM has specific advantage in treatment of gastric ulcer, especially for chronic and recurrent gastric ulcer, which was associated with relieving gastric ulcer burning pain symptom, suppressing gastric ulcer mucosal inflammation, inhibiting gastric ulcer mucosal oxidative stress, improving gastric ulcer mucosal microenvironment.

\section{Sources and funding}

This study supported by grant $(81741007,81870363,81700395$ and 81870217) from National Natural Science Foundation of China; Grant 2020JDTD0025 and 2020JDTD0022 from science \& technology departments of Sichuan province. And grant 008066, 030038199, 030041023, 030041224 and 242030016 from Cheng Du University of Traditional Chinese Medicine.

\section{Reference}

1. Pawlik T (2002) Impact of Helicobacter pylori and nonsteroidal anti-inflammatory drugs on gastric ulcerogenesis in experimental animals and in humans. Eur $J$ Pharmacol 449: 1-15.

2. Tabiri S, Akanbong P, Abubakari BB (2016) Assessment of the environmental risk factors for a gastric ulcer in northern Ghana. Pan Afr Med J 25: 160.

3. Tarnawski AS, Ahluwalia A, Jones MK (2014) Increased susceptibility of aging gastric mucosa to injury: the mechanisms and clinical implications. World J Gastroenterol 20: 4467-4482. [Crossref]

4. Liu ZY (2018) Effects of polaprezinc on gastric mucosal damage and neurotransmitters in a rat model of chemotherapy-induced vomiting. J Int Med Res 46: 2436-2444. [Crossref]

5. Kawanishi Y (1997) Synthesis and biological evaluation of a new reversely linked type of dual histamine H2 and gastrin receptor antagonist. Chem Pharm Bull 45: 116-124.

6. Satoh H, Akiba Y, Urushidani T (2020) Proton Pump Inhibitors Prevent Gastric Antra Ulcers Induced by NSAIDs via Activation of Capsaicin-Sensitive Afferent Nerves in Mice. Dig Dis Sci 65: 2580-2594. [Crossref]

7. Ko IG (2018) Combination therapy with polydeoxyribonucleotide and proton pump inhibitor enhances therapeutic effectiveness for gastric ulcer in rats. Life Sci 203: 1219. [Crossref]

8. He P (2015) Curcumin-induced histone acetylation inhibition improves stress-induced gastric ulcer disease in rats. Mol Med Rep 11: 1911-1916. [Crossref]

9. Hojgaard L, Mertz Nielsen A, Rune SJ (1996) Peptic ulcer pathophysiology: acid, bicarbonate, and mucosal function. Scand J Gastroenterol Suppl 216: 10-15. [Crossref]

10. Tulassay Z, Herszenyi L (2010) Gastric mucosal defense and cytoprotection. Best Pract Res Clin Gastroenterol 24: 99-108.

11. Najm WI (2011) Peptic ulcer disease. Prim Care 38: 383-394.

12. Kato I, Nomura AM, Stemmermann GN, Chyou PH (1992) A prospective study of gastric and duodenal ulcer and its relation to smoking, alcohol, and diet. Am J Epidemiol 135: 521-530. [Crossref]

13. Ajayi AF, Olaleye BS (2020) Age-related changes in haematological parameters and biochemical markers of healing in the stomach of rats with acetic acid induced injury. Toxicol Rep 7: 1272-1281. [Crossref]

14. Alp MH, Court JH, Grant AK (1970) Personality pattern and emotional stress in the genesis of gastric ulcer. Gut 11: 773-777. [Crossref]

15. Kucukazman M, Yeniova O, Dal K, Yavuz B (2015) Helicobacter pylori and cardiovascular disease. Eur Rev Med Pharmacol Sci 19: 3731-3741. [Crossref]

16. Rubio CA, Namiki H, Stemmermann G (1993) Geographic variations in the histological characteristics of the gastric mucosa. J Environ Pathol Toxicol Oncol 12: 89-92.

17. Graham DY (2014) History of Helicobacter pylori, duodenal ulcer, gastric ulcer and gastric cancer. World J Gastroenterol 20: 5191-5204. [Crossref]

18. Wang AY, Peura DA (2011) The prevalence and incidence of Helicobacter pyloriassociated peptic ulcer disease and upper gastrointestinal bleeding throughout the world. Gastrointest Endosc Clin N Am 21: 613-635.
19. Warren JR, Marshall B (1983) Unidentified curved bacilli on gastric epithelium in active chronic gastritis. Lancet 1: 1273-1275. [Crossref]

20. Lanas A, Chan FKL (2017) Peptic ulcer disease. Lancet 390: 613-624.

21. Perini R, Fiorucci S, Wallace JL (2004) Mechanisms of nonsteroidal anti-inflammatory drug-induced gastrointestinal injury and repair: a window of opportunity for cyclooxygenase-inhibiting nitric oxide donors. Can J Gastroenterol 18: 229-236.

22. Mabrok HB, Mohamed MS (2019) Induction of COX-1, suppression of COX-2 and pro-inflammatory cytokines gene expression by moringa leaves and its aqueous extract in aspirin-induced gastric ulcer rats. Mol Biol Rep 46: 4213-4224. [Crossref]

23. Motilva V, Lastra CADL, Bruseghini L, Herrerias JM, Fidalgo SS (1997) COX expression and PGE(2) and PGD(2) production in experimental acute and chronic gastric lesions. Int Immunopharmacol 5: 369-379. [Crossref]

24. Desai JK, Goyal RK, Parmar NS (1997) Pathogenesis of peptic ulcer disease and current trends in therapy. Indian J Physiol Pharmacol 41: 3-15.

25. Adeniyi OS, Emikpe BO, Olaleye SB (2018) Accelerated gastric ulcer healing in thyroxine-treated rats: roles of gastric acid, mucus, and inflammatory response. Can J Physiol Pharmacol 96: 597-602. [Crossref]

26. Takeuchi K (2012) Pathogenesis of NSAID-induced gastric damage: importance of cyclooxygenase inhibition and gastric hypermotility. World J Gastroenterol 18: 2147 2160 .

27. Werther JL (2000) The gastric mucosal barrier. Mt Sinai J Med 67: 41-53. [Crossref]

28. Yao H (2019) Andrographolide attenuates imbalance of gastric vascular homeostasis induced by ethanol through glycolysis pathway. Sci Rep 21: 4968. [Crossref]

29. Salam OMA, Czimmer J, Debreceni A, Szolcsanyi J, Mozsik G (2001) Gastric mucosal integrity: gastric mucosal blood flow and microcirculation. An overview. $J$ Physio Paris 95: 105-127. [Crossref]

30. Michiels C (2003) Endothelial cell functions. J Cell Physiol 196: 430-443. [Crossref]

31. Mehta D, Malik AB (2006) Signaling mechanisms regulating endothelial permeability Physiol Rev 86: 279-367. [Crossref]

32. Magierowska K (2019) Time-dependent course of gastric ulcer healing and molecular markers profile modulated by increased gastric mucosal content of carbon monoxide released from its pharmacological donor. Biochem Pharmacol 163: 71-83. [Crossref]

33. Baraka AM, Guemei A, Gawad HA (2010) Role of modulation of vascular endothelia growth factor and tumor necrosis factor-alpha in gastric ulcer healing in diabetic rats. Biochem Pharmacol 79: 1634-1639. [Crossref]

34. Costa AM (20(16) Helicobacter pylori Activates Matrix Metalloproteinase 10 in Gastric Epithelial Cells via EGFR and ERK-mediated Pathways. J Infect Dis 213: 1767-1776. [Crossref]

35. Swarnakar S (2005) Curcumin regulates expression and activity of matrix metalloproteinases 9 and 2 during prevention and healing of indomethacin-induced gastric ulcer. J Biol Chem 280: 9409-9415. [Crossref]

36. Teschke R, Wolff A, Frenzel C, Eickhoff A, Schulze J (2015) Herbal traditional Chinese medicine and its evidence base in gastrointestinal disorders. World J Gastroenterol 21: 4466-4490. [Crossref]

37. Hu L, Li HY, Chen WQ, Lao SX, Luo Q (2019) Ultrastructure Characteristics of Different Chinese Medicine Syndromes of Helicobacter pylori-Correlated Gastric Diseases. Chin J Integr Med 25: 917-921. [Crossref]

38. Yi L, Lu Y, Yu S, Cheng Q, Yi L (2020) Formononetin inhibits inflammation and promotes gastric mucosal angiogenesis in gastric ulcer rats through regulating NF- $\mathrm{\kappa B}$ signaling pathway. J Recept Signal Transduct Res 26.

39. Lou G (2020) Tanshinone II A attenuates vascular remodeling through klf4 mediated smooth muscle cell phenotypic switching. Sci Rep 10: 13858. [Crossref]

40. Ren S (2020) Rutaecarpine Ameliorates Ethanol-Induced Gastric Mucosal Injury in Mice by Modulating Genes Related to Inflammation, Oxidative Stress and Apoptosis. Front Pharmacol 11: 600295.

Copyright: (C2021 Huan Xu. This is an open-access article distributed under the terms of the Creative Commons Attribution License, which permits unrestricted use, distribution, and reproduction in any medium, provided the original author and source are credited. 